\title{
Osteogenic potential of primary stem cells derived from the human dental pulp is enhanced by carboxymethyl cellulose/chitosan scaffold doped with wollastonite particles
}

\author{
Dannie Macrin $^{1}$ (D), Vivek Narayanan ${ }^{2}$, Arikketh Devi ${ }^{3 *}$ \\ ${ }^{1}$ Department of Computational Biology, Institute of Bioinformatics, Saveetha School of Engineering, SIMATS Saveetha Institute of Medical and Technical \\ Sciences, Chennai, India. \\ ${ }^{2}$ Department of Oral and Maxillofacial Surgery, SRM Kattankulathur Dental College, SRM Institute of Science and Technology, Chennai, India. \\ ${ }^{3}$ Cancer and Stem Cell Biology Laboratory, Department of Genetic Engineering, SRM Institute of Science and Technology, Kattankulathur, India.
}

\begin{tabular}{l}
\hline ARTICLE INFO \\
\hline Article history: \\
Received on: January 18, 2021 \\
Accepted on: June 03, 2021 \\
Available online: September 20, 2021 \\
\hline
\end{tabular}

\section{Key words:}

Adult stem cells, mesenchymal markers, bone regeneration, osteogenic differentiation

\begin{abstract}
The success of any scaffold assisted regenerative therapy relies on two major factors; a conducive scaffold material and versatile, viable stem cells. In our study, we suggest chitosan (CS)/carboxymethylcellulose (CMC) scaffold functionalized with wollastonite (WS) $\left(\mathrm{CaSiO}_{3}\right)$ particles as a conducive scaffold material which mimics the porous structure of the bone and promotes osteogenesis. We have paired this scaffold material with stem cells derived from dental pulp (DPSCs) which is an ideal cell source with a high percentage of stem cells and have a natural inclination toward forming hard calcified tissues. In our experiments, the cells isolated from human dental pulp produced a cell population with high percentage of cells positive for mesenchymal markers CD73 and CD90. The DPSCs were able to differentiate into osteoblasts when induced with media supplemented with $\beta$-glycerophosphate, ascorbic acid, and dexamethasone. Furthermore, physicochemical analysis of CS/CMC/WS scaffold showed that it formed a highly porous structure conducive to cell growth, penetration, and nutrient uptake. Furthermore, $\mathrm{CS} / \mathrm{CMC} / \mathrm{WS}$ scaffold promoted osteogenic differentiation of DPSCs. We propose CS/CMS/WS scaffold paired with DPSCs as an effective system for bone regeneration.
\end{abstract}

\section{INTRODUCTION}

Adult stem cells are present in almost all tissues of the human body, performing the primary function of maintaining and repairing the tissues in which they are residing. They are known to inhabit specific niches and has the potential to self-renew and differentiate into more functional cell types. In the past decade, an increasing number of studies tried to exploit the abilities of adult stem cells for regenerative medicine. Mesenchymal stem cells (MSCs) also known as skeletal stem cells is one of the most predominantly present stem cells in an adult human body. This progenitor cell population has the ability to give rise to at least three of the mesodermal tissue types such as the chondrocytes, osteoblasts, and adipocytes even in in vitro conditions [1]. MSCs were first isolated from bone marrow mononuclear cells and later

*Corresponding Author

Arikketh Devi, Cancer and Stem Cell Biology Laboratory, Department

of Genetic Engineering, SRM Institute of Science and Technology,

Kattankulathur, India.E-mail: devia@srmist.edu.in found to be present in multiple sites across the human body [2]. Interestingly, the human dental pulp was identified as one of such sites which possessed niches for MSCs.

Stanley et al. [3] reported cells inside the dental pulp which not only were able to undergo mitosis but also were able to differentiate into immature preodontoblasts which later gave rise to odontoblasts. In the early 21 st century, further molecular and cellular level analysis of these cells proved their stem cell-like properties. Gronthos et al. [4] isolated stromal cell population from dental pulp transplants and were able to differentiate them into adipogenic and neurogenic lineages. Immunolocalization studies revealed a niche of stromal antigen $1\left(\mathrm{STRO} 1^{+}\right.$) MSCs in the perivascular and perineural spaces of the dental pulp [5]. Developmentally, dental pulp cells are derived from ectodermal tissues from the periphery of the neural tube which migrate to the oral region. For this reason, dental pulp cells are also termed as "ectomesenchyme" [6]. Dental pulp stem cells (DPSCs) have a similar immunophenotype compared to the bone marrow-mesenchymal stem cells, and they are known to express surface antigens CD44 (homing cell adhesion molecule), 
CD29 (integrin subunit beta 1), CD73 (5NTD, 5'-nucleotidase ecto), CD146 (melanoma cell adhesion molecule), CD271 (nerve growth factor), and Strol [7-9]. Apart from the three mesodermal lineages, DPSCs are also shown to transdifferentiate into other cell types such as keratocytes of the corneal stroma [10], neuronlike cells [11]. DPSCs contribute to regeneration not only by differentiation, they also secrete angiogenic related molecules such as endostatin, insulin like growth factor binding protein 3 , interleukin 8 (IL8) (chemokine ligand 8), monocyte chemoattractant protein 1 (chemokine ligand 2), tissue inhibitor of metalloproteinase 1, vascular endothelial growth factor [12], and beneficial factors such as leptin, fractalkine, neutrophil activating peptide-2, macrophage inflammatory protein-1b (MIP-1b), and MIP-3a, as well as a small number of cytokines (IL-6, -7, -8, and -10 ), growth factors (granulocyte-colony stimulating factor, macrophage colony stimulating factor, granulocyte macrophage colony stimulating factor, stromal derived factor 1 , and stem cell factor) [13]. The use of these adult MSCs in regenerative therapy of larger defects like bone fracture is facilitated by their ability to proliferate on a scaffold material which localizes the MSCs on the site of the defect and may secrete beneficial factors that enhances their proliferation and differentiation.

Scaffolds are 3D porous materials which act as a template for tissue formation, and they are usually seeded with cells and occasionally "doped" with growth or differentiation factors [14]. Efficient scaffold designing should create appropriate porous structures to ensure desired mechanical function and mass transport properties. This along with the material chemistry of the scaffold determines the maximum functional properties that a scaffold can achieve, as well as how cells interact with the scaffold [15]. Bone tissue engineering (BTE) is one of the avenues of regenerative medicine where scaffolds are widely used to overcome the lack of bone grafts traditionally used in the treatment of critically sized bone defects. [16]. The bio-scaffolds used for BTE should be biocompatible, biodegradable and mimic in vitro extracellular matrix [17]. Chitosan (CS) is a linear cationic polymer which is composed of partially deacetylated chitin, glycosamine, and N-Acetyl glycosamine with $\beta$ linkage [18]. Carboxymethylcellulose (CMC) is an anionic polymer derived from cellulose which has a similar structure to $\mathrm{CS}$, and hence establishes a strong ionic crosslinkage with CS. This results in a polyelectrolytic, hydrophilic, protein absorbing complex [19]. Wollastonite (WS) is a naturally occurring mesoporous calcium silicate particle which was shown to be biodegradable and bioactive [20]. WS enhances the differentiation of human bone marrow stromal cells to differentiate toward osteoblasts. This stimulatory effect is caused by the release of ionic products released from the WS particle into the culture medium [20]. C3H10T1/2 murine mesenchymal cells treated with $\mathrm{CS}+\mathrm{CMC}+\mathrm{WS}$ scaffold conditioned medium revealed that $\mathrm{CS}+$ $\mathrm{CMC}+\mathrm{WS}$ exhibits osteoconductive effect when used along with an osteogenic medium (OM) [21].

\section{MATERIALS AND METHODS}

\subsection{Dental Pulp Isolation and Primary Culture}

Teeth (premolars) that were isolated for orthodontic purposes were obtained from SRM Dental College, Potheri, Tamil Nadu, India
(Institutional Ethical Clearance Number: 835/IEC/2015). In this study, we have used cells from two male patients of 28 and 22 years of age which were labeled as DPSC 01 and DPSC 02, respectively. The extracted teeth were immediately immersed in DMEM (Dulbeccos modified eagle's medium) Glutamax ${ }^{\circledR}$ media (Gibco) with $10 \%$ foetal bovine serum (Gibco) in a sterile centrifuge tube and stored at $4{ }^{\circ} \mathrm{C}$. The teeth were collected and transported back to the lab within 24 hours. In the lab, the individual tooth was cut mechanically using a rotary saw at the cementoenamel junction and the pulp was extracted. The pulp tissue was minced into chunks with a sterile scalpel. The tissue chunks were then enzymatically digested with a solution of collagenase $(12 \mathrm{mg} / \mathrm{ml})$ and dispase (16 $\mathrm{mg} / \mathrm{ml})$ at $37^{\circ} \mathrm{C}$ for 1 hour. After digestion, the enzyme + tissue mixture was centrifuged at 2,000 RPM for 10 minutes at $4{ }^{\circ} \mathrm{C}$. The pellet was re-suspended in DMEM Glutamax ${ }^{\circledR}$ media $+10 \%$ FBS $+1 \times$ antibiotic-antimycotic solution (Sigma-Aldrich) and seeded in T25 filtered culture flask and incubated at $37^{\circ} \mathrm{C}$ with $5 \% \mathrm{CO}_{2}$. The media was changed every 3 days until the cells egressed out of the tissues and formed a confluent monolayer.

\subsection{Cytometric Analysis}

Confluent DPSC 01 and DPSC 02 were trypsinized with $0.25 \%$ Trypsin + ethylene diamine tetra acetic acid reagent (T4049, Sigma-Aldrich) and triturated until no clumps are present. The DPSCs were fixed with $4 \%$ paraformaldehyde in phosphate buffer saline (PBS) for 20 minutes over ice. Primary antibodies CD90 (sc-53116), CD73 (sc-398260) or CD34 (sc-74499) were used. The primary stained cells were washed after 1 hour and incubated with a fluorescent secondary antibody (AlexaFlour 488, ThermoFisher scientific) for 20 minutes. The stained cells were analyzed in Cytomics FC-500 (Beckman-Coulter) with CXP software.

\subsection{Scaffold Preparation}

The CS/CMC/WS scaffold was prepared as previously described by Saravanan et al. [22]. 1\% (W/V) CS was added to 1\% (W/V) CMC solubilized in distilled water. To this, $1 \%(\mathrm{~W} / \mathrm{V}) \mathrm{WS}$ was added and stirred continuously to obtain a homogeneous mixture. This mixture was cast into 24 well plates and lyophilized. The scaffold material was neutralized with $1 \mathrm{M} \mathrm{NaOH}$ and washed repeatedly with distilled water.

\subsection{Physicochemical Characterization of $\mathrm{CS} / \mathrm{CMC} / \mathrm{WS}$ Scaffolds}

The surface morphology of the lyophilized CS/CMC/m-WS scaffolds were examined and assessed by scanning electron microscopy (SEM) and transmission electron microscope (TEM). SEM analysis was performed in a Quanta FEI 250 instrument (FEI, OR). A small piece of the scaffold was mounted on a "STUB" and loaded in the sample drawer. High magnification images were obtained with (low vacuum) condition. The pore size of multiple pores was measured using the Tecnai ${ }^{\circledR}$ software.

TEM imaging and energy dispersive analysis of $\mathrm{X}$ rays were performed in an FEI Tecnai TEM ${ }^{\circledR}$ machine. The scaffold powder was placed in a sonic bath and then few drops of the welldispersed solution were placed in a "MATRIX DOT" and dried. 

carboxymethyl cellulose/chitosan scaffold doped with wollastonite particles 2021;9(S1):1-6

The "MATRIX DOT" was then placed on the sample holder of the TEM machine.

\subsection{Osteogenic Differentiation and Alizarin Red Staining}

The DPSC 02 cells were cultured in a 6-well plate until they were $\sim 60 \%$ confluent. $1 \mathrm{ml}$ of osteogenic media comprising base media, $10 \%$ FBS, $10 \mathrm{mM} \beta$-Glycerophosphate, $0.2 \mathrm{mM}$ L-Ascorbic acid and $100 \mathrm{nM}$ Dexamethasone was added over the cells. For conditioned osteogenic medium (C-OM), the media described above was mixed with $5 \mathrm{mg} / \mathrm{ml}$ of lyophilized scaffold powder and incubated at $4^{\circ} \mathrm{C}$ for 48 hours. The cells were incubated in $\mathrm{OM}$ for a time period of 10 days with media change every 3 days. After the stipulated time, the cells were fixed with $4 \%$ formaldehyde and stained with Alizarin red stain and incubated for 10 minutes. The excess stain was washed off with PBS and visualized under a microscope. For spectrometric quantification, the stain was released with $10 \%$ acetic acid and neutralized with $0.1 \mathrm{M}$ ammonium hydroxide. The absorbance was measured at $405 \mathrm{~nm}$.

\subsection{Confocal Analysis}

The differentiated osteoblasts were surface seeded on the precasted scaffold in a 24 well plate. The plates were incubated at $37^{\circ} \mathrm{C}$ with $5 \% \mathrm{CO}_{2}$ for a period of 7 days. The scaffold material was then sliced transversely and the slices were stained with NucBlue ${ }^{\circledR}$
Live Cell stain (Life Technologies). They were then mounted on a slide and visualized under confocal microscopy system (SP5, Leica). The stain retained by the scaffold was negated by masking with a green channel over the scaffold auto-fluorescence.

\section{RESULTS AND DISCUSSION}

\subsection{The Primary Cells Obtained from Human Dental Pulp Expressed MSC Markers}

The cells which egressed from the enzymatically digested tissue chunks eventually formed colonies and then developed into a monolayer (Fig. 1A). The cells showed uniform fibroblastic morphology and were able to proliferate at a rapid rate. These cells were expanded and after two passages the cells were subjected to cytometric analysis for known mesenchymal markers CD90 (Thy1) and CD73 (nucleotidase/ ecto-5-nucleotidase) [23,24] and CD34, a hematopoietic antigen was used as a negative marker. The DPSCs derived from two different donors had a large population of CD90+ (79.3\%, 96.1\%) and CD73+ (99.4\%, 98.9\%) cells (Fig. 1B).

\subsection{CS/CMC/WS Scaffold Formed a Highly Porous Surface Conducive to Cell Growth and Nutrient Absorption}

Uniformly sized spherical mesoporous WS particles (Fig. 2A) were mixed with $\mathrm{CS}$ and $\mathrm{CMC}$ to form the $\mathrm{CS} / \mathrm{CMC} / \mathrm{WS}$ scaffold. The

\section{$\mathbf{A}$}
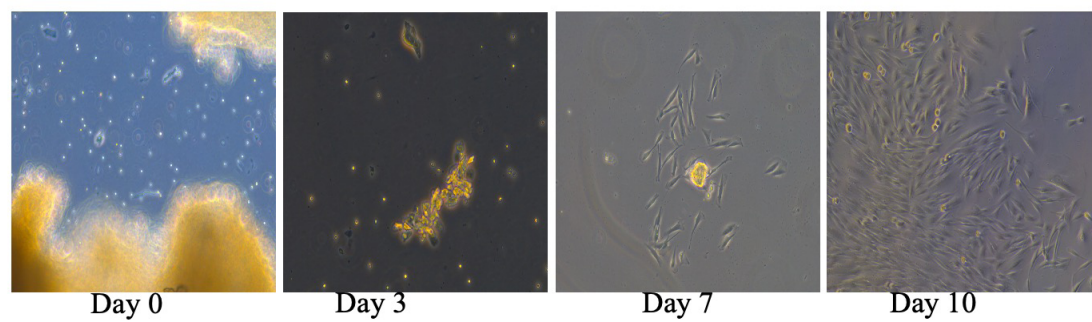

B
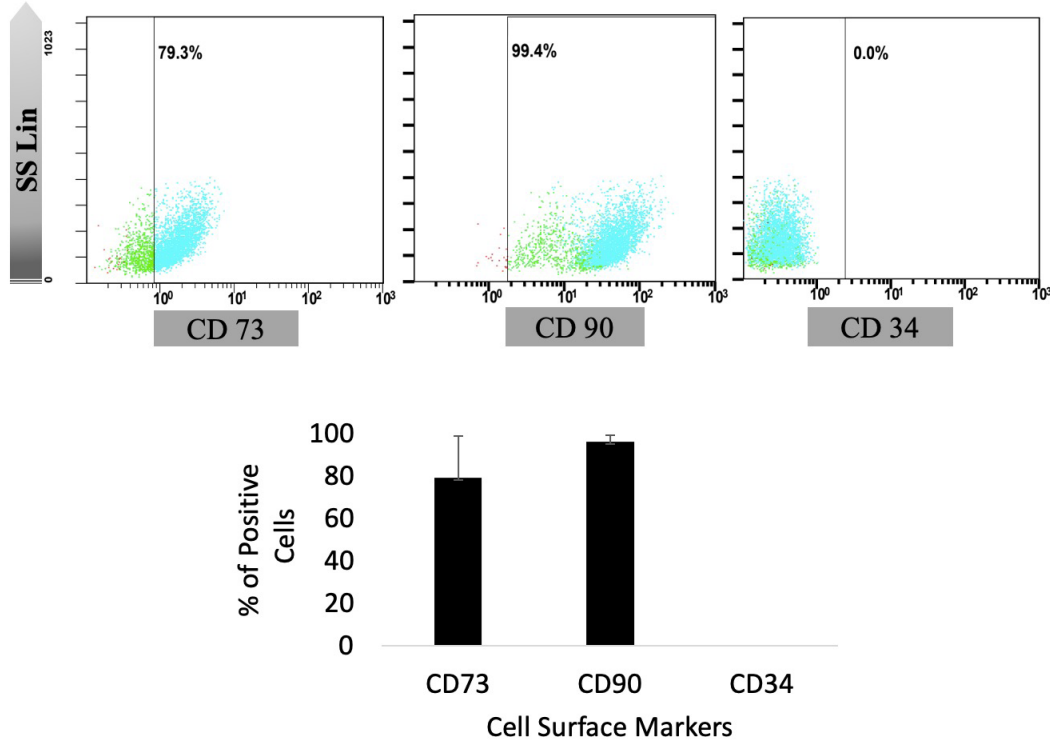

Figure 1: Primary cells from human dental pulp express mesenchymal markers. (A) Representative micro pictographs of cells egressing from enzyme-treated dental pulp tissue. (B) Fluorescence assisted cytometry of DPSC lines from two different donors showed a high population of CD73+ and CD90+ cells. CD34 was used as a negative marker. 
$\mathbf{A}$
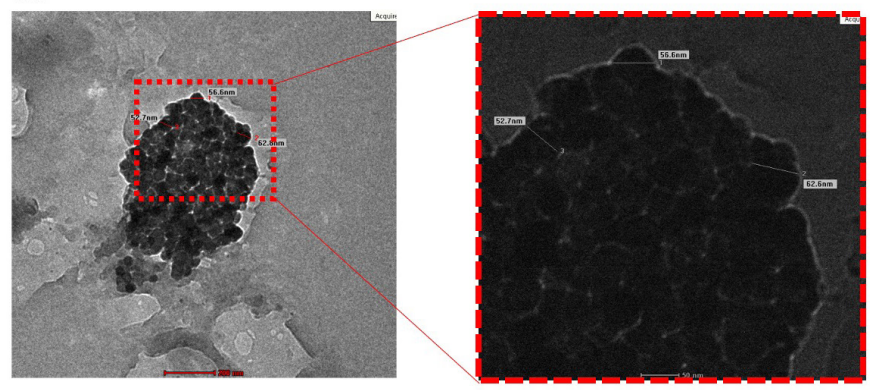

B

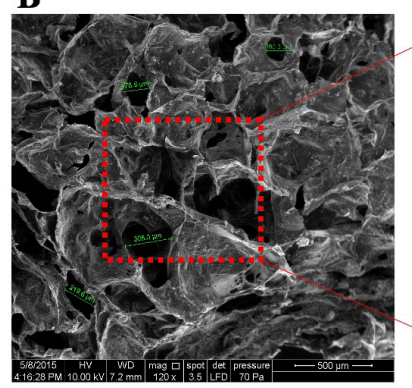

C
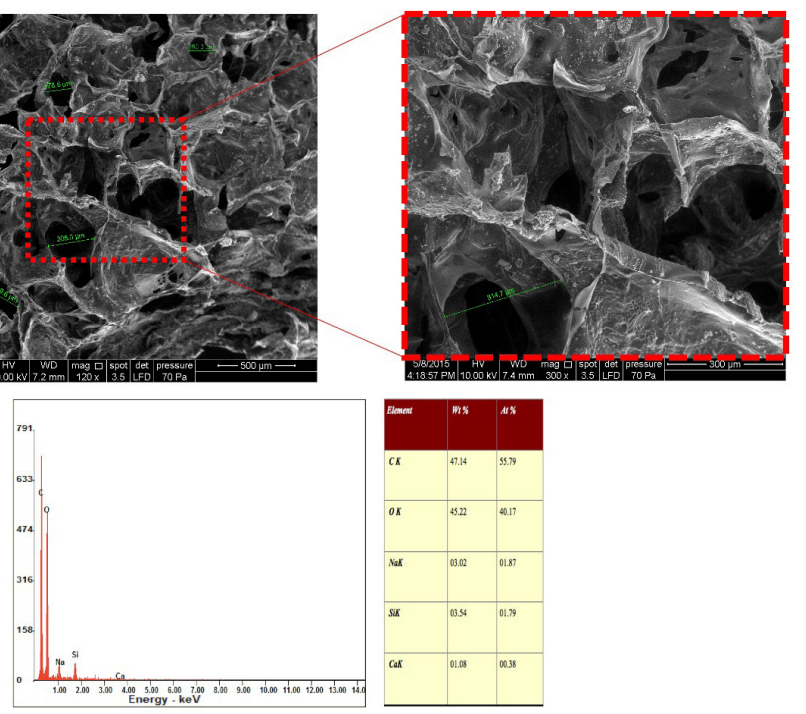

Figure 2: Physiochemical characterization of CS/CMC/WS scaffold. (A) TEM micro pictograph of WS crystals revealed the spherical architecture of the particles. (B) SEM micro pictograph of $\mathrm{CS} / \mathrm{CMC} / \mathrm{WS}$ scaffold demonstrated a highly porous structure with average pore size $215 \mu \mathrm{m}$. (C) EDX analysis of $\mathrm{CS} / \mathrm{CMC} / \mathrm{WS}$ scaffold revealed the atomic composition of $\mathrm{Ca}, \mathrm{Si}, \mathrm{Na}, \mathrm{C}$, and $\mathrm{O}$.

surface morphology of the CS/CMC/WS scaffold was dissected using SEM. The pore size of the lyophilized scaffold ranged from 160.3 to $314.7 \mu \mathrm{m}$ (Fig. 2B) which was reported optimal for cell attachment, proliferation, and migration [25]. The energy dispersive X-ray (EDX) analysis revealed the constituent elements of the CS/CMC/WS scaffold: carbon (C), oxygen $(\mathrm{O})$, sodium $(\mathrm{Na})$, silica (Si), and calcium (Ca) (Fig. 2C). Previous studies indicated that the silanol (Si-OH) group enhanced the attachment of organic functional groups on its surface [26]. Adding to this, the mesoporous surface provided an increased surface area with $-\mathrm{OH}$ and $\mathrm{Si}-\mathrm{OH}$ which facilitated adsorption [27].

\subsection{Exudates from the CS/CMC/WS Scaffold Facilitated Osteogenic Differentiation of DPSCs}

The DPSCs were subjected to osteogenic differentiation for a period of 10 days. After this time period, extracellular calcifications were observed in the cells that were treated with OM and scaffold C-OM. These calcifications were visualized by staining with alizarin red. It was observed that the cells treated with C-OM showed increased extracellular calcifications compared to the cells treated with unconditioned OM (Fig. 3A). The spectrophotometric analysis of

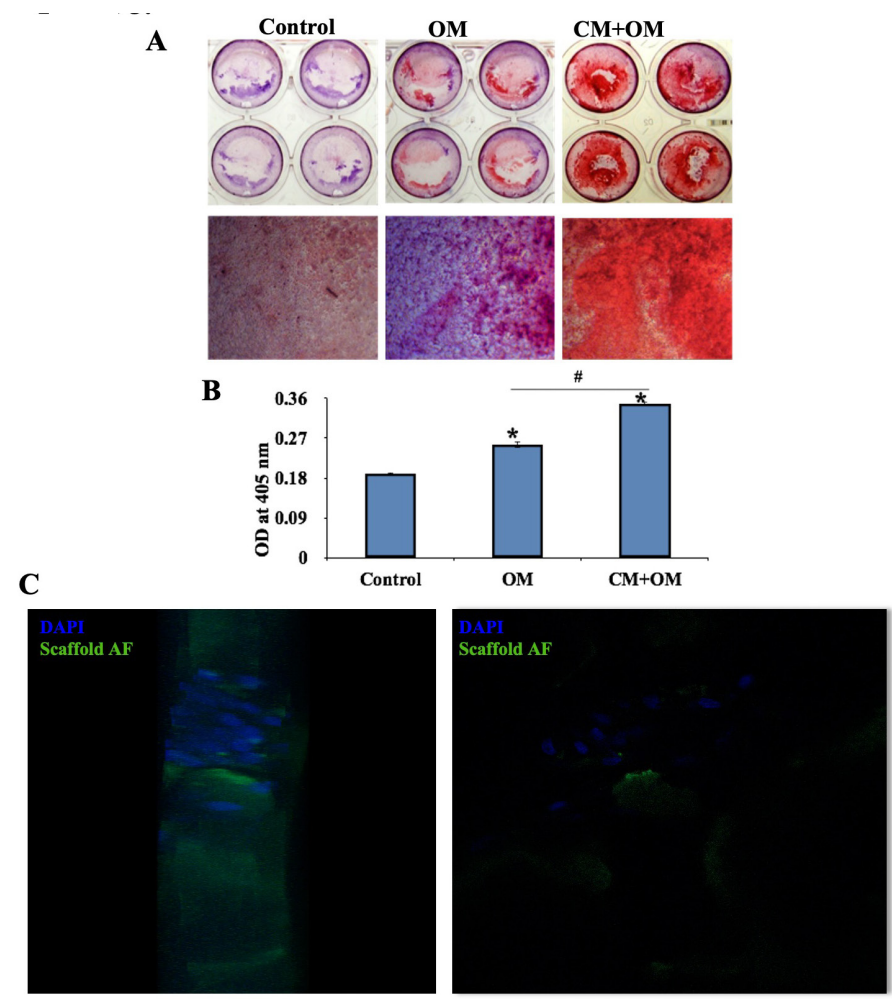

Figure 3: CS/CMC/WS scaffold facilitates osteodifferentiation of DPSCs. DPSCs were grown in the presence and absence of CS/CMC/WS scaffold $\mathrm{C}-\mathrm{OM}$ (CM + OM). (A) The highest calcification was observed in DPSCs treated with $(\mathrm{CM}+\mathrm{OM})$. (B) Quantification plots of calcification control, $\mathrm{OM}, \mathrm{CM}+\mathrm{OM}$ treated cells. (C) Confocal micro pictographs of osteoblasts differentiated from DPSCs forming colonies within the scaffold structure.

the alizarin dye released from these samples demonstrated that the C-OM threated samples absorbed $\sim 1.5$ times more alizarin than the OM treated samples. This indicated that the $\mathrm{CS} / \mathrm{CMC} / \mathrm{WS}$ scaffold was osteoconductive. These results were congruent with the studies conducted on mouse MSCs [21,22].

\subsection{DPSCs were Able to Penetrate and Colonize the CS/ CMC/WS Scaffold}

Osteoblasts were derived from the DPSCs using the abovementioned protocol. These osteoblasts were then trypsinized and seeded over the scaffold surface which was precast in a 24well plate. The cells were allowed to grow for a period of 7 days supplemented with the OM. At the end of this period, the scaffold material was thinly sliced using a sterile scalpel and mounted on a glass side and visualized under the confocal microscope. The transverse sectioning revealed multiple colonies of DPSC derived osteoblasts localized within the scaffold material. This suggests that the porosity of the $\mathrm{CS} / \mathrm{CMC} / \mathrm{WS}$ scaffold permitted cell proliferation and penetration.

\section{CONCLUSION}

From the results of our experiments, we conclude that a large percentage of cells isolated from human dental pulp were CD73 and CD 90 positive MSCs which were capable of differentiating into 
carboxymethyl cellulose/chitosan scaffold doped with wollastonite particles 2021;9(S1):1-6

osteoblasts when induced by media supplements. The mesoporous $\mathrm{CS} / \mathrm{CMC} / \mathrm{WS}$ scaffolds provide a highly porous surface conducive to cell proliferation, penetration and nutrient absorption. The CS/ CMC/WS scaffold also secretes soluble exudates which enhances the osteogenic differentiation of the human dental pulp-derived stem cells. Studies have also proved $\mathrm{CS} / \mathrm{CMC} / \mathrm{WS}$ scaffolds to be bioactive and non-toxic [21]. Based on these conclusions, we propose DPSCs seeded on CS/CMC/WS scaffold material as a viable system for regenerative therapy of bone defects.

\section{LIST O F ABBREVIATIONS}

BTE, Bone tissue engineering; C-OM, Conditioned osteogenic medium; CMC, Carboxymethylcellulose; CS, Chitosan; DMEM, Dulbeccos modified eagle's medium; DPSC, Dental pulp stem cells; EDX, Energy dispersive X-ray; IL 8, Interleukin 8; MSCs, Mesenchymal stem cells; OM, Osteogenic medium; PBS, Phosphate buffer saline; SEM, Scanning electron microscope; Stro 1, Stromal antigen 1; TEM, Transmission electron microscope; WS, Wollastonite.

\section{ETHICS APPROVAL}

All procedures followed were in accordance with the ethical standards of the responsible committee on human experimentation (SRM Medical College Hospital and Research Centre, Clearance Number: 835/IEC/2015) and with the Helsinki Declaration of 1975 , as revised in 2008 .

\section{CONSENT TO PARTICIPATE}

Informed consent was obtained from all participants for being included in the study.

\section{CONSENT FOR PUBLICATION}

No individual patient information was used.

\section{CONFLICT OF INTEREST}

The authors declare that we have no conflict of interest.

\section{FUNDING}

No external funding was obtained for this study.

\section{AUTHOR CONTRIBUTIONS}

Arriketh Devi and Macrin D conceived the idea and developed the hypothesis. Macrin D performed the experiments, interpreted the results, and prepared the manuscript.

Selvamurugan $\mathrm{N}$ provided materials and methods required for scaffold preparation and provided intellectual support in the physicochemical analysis of the synthesized scaffold material.

Narayanan V provided the samples required for the research and provided support in extraction of the pulp from the teeth.

Devi A, Selvamurugan N, and Narayanan V supervised and helped in drafting the manuscript.

\section{ACKNOWLEDGMENTS}

The authors would like to thank Dr. Lakshmi Rathan, SRM Kattankulathur Dental College for performing the extraction of teeth from patients.

\section{REFERENCES}

1. Sheng G. The developmental basis of mesenchymal stem/stromal cells (MSCs). BMC Dev Biol 2015;15:44; doi:10.1186/s12861-015-00945.

2. Lv FJ, Tuan RS, Cheung KM, Leung VY. Concise review: the surface markers and identity of human mesenchymal stem cells. Stem Cells 2014;32:1408-19; doi:10.1002/stem.1681.

3. Stanley HR. The cells of the dental pulp. Oral Surg Oral Med Oral Pathol 1962;15:849-58; doi:10.1016/0030-4220(62)90337-7.

4. Gronthos S, Brahim J, Li W, Fisher LW, Cherman N, Boyde A, et al. Stem cell properties of human dental pulp stem cells. J Dent Res 2002;81:531-5; doi:10.1177/154405910208100806.

5. Shi S, Gronthos S. Perivascular niche of postnatal mesenchymal stem cells in human bone marrow and dental pulp. J Bone Miner Res 2003;18:696-704; doi:10.1359/jbmr.2003.18.4.696.

6. Chang CC, Chang KC, Tsai SJ, Chang HH, Lin CP. Neurogenic differentiation of dental pulp stem cells to neuron-like cells in dopaminergic and motor neuronal inductive media. J Formos Med Assoc 2014;113:956-65; doi:10.1016/j.jfma.2014.09.003.

7. Kawashima N. Characterisation of dental pulp stem cells: a new horizon for tissue regeneration? Arch Oral Biol 2012;57:1439-58; doi:10.1016/j.archoralbio.2012.08.010.

8. Ledesma-Martínez E, Mendoza-Núñez VM, Santiago-Osorio E. Mesenchymal stem cells derived from dental pulp: a review. Stem Cells Int 2016;2016: 4709572. Available via http:/ www.embase.com/search/results? subaction=viewrecord \&from=export\&id=L $607447812 \% 5 \mathrm{Cnhttp}: / / \mathrm{dx}$.doi. org/10.1155/2016/4709572\%5Cnhttp://1imo.1ibis.be/ resolver?\&sid=EMBASE\&issn=16879678\&id=doi:10.1155/201 6/4709572\&atitle=Mesenchymal+stem+cells+derived+from.

9. Sivasankar V, Ranganathan K. Growth characteristics and expression of CD73 and CD146 in cells cultured from dental pulp. J Investig Clin Dent 2016;7:278-85; doi:10.1111/jicd.12155.

10. Syed-Picard FN, Du Y, Lathrop KL, Mann MM, Funderburgh ML, Funderburgh JL. Dental pulp stem cells: a new cellular resource for corneal stromal regeneration. Stem Cells Transl Med 2015;4:276-85; doi:10.5966/sctm.2014-0115.

11. Chun SY, Soker S, Jang YJ, Kwon TG, Yoo ES. Differentiation of human dental pulp stem cells into dopaminergic neuron-like cells in vitro. J Korean Med Sci 2016;31:171-7; doi:10.3346/ jkms.2016.31.2.171.

12. Bronckaers A, Hilkens P, Fanton Y, Struys T, Gervois P, Politis C, et al. Angiogenic properties of human dental pulp stem cells. PLoS One 2013;8:e71104; doi:10.1371/journal.pone.0071104.

13. Nakashima M, Iohara K, Murakami M. Dental pulp stem cells and regeneration. Endod Topics 2013;28:38-50; doi:10.1016/S00766879(06)19005-9.

14. O'brien FJ. Biomaterials \& scaffolds for tissue engineering. Mater Today 2011;14:88-95; doi:10.1016/S1369-7021(11)70058-X.

15. Hollister SJ. Porous scaffold design for tissue engineering. Nat Mater 2005;4:518-24; doi:org/10.1038/nmat1421.

16. Petrovic V, Zivkovic P, Petrovic D, Stefanovic V. Craniofacial bone tissue engineering. Oral Surg Oral Med Oral Pathol Oral Radiol 2012;114:e1-9; doi:10.1016/j.oooo.2012.02.030.

17. Rezwan K, Chen QZ, Blaker JJ, Boccaccini AR. Biodegradable and bioactive porous polymer/inorganic composite scaffolds for bone tissue engineering. Biomaterials 2006;27:3413-31; doi:10.1016/j. biomaterials.2006.01.039. 
18. Knorr D. Functional properties of chitin and chitosan. J Food Sci 1982;47:593-5; doi:10.1111/j.1365-2621.1982.tb10131.x.

19. Fei Liu X, Lin Guan Y, Zhi Yang D, Li Z, De Yao K. Antibacterial action of chitosan and carboxymethylated chitosan. J Appl Polym Sci 2001;79:1324-35; doi:10.1002/1097-4628(20010214)79:7<1324::AIDAPP210>3.0.CO;2-L.

20. Li H, Zhai W, Chang J. Effects of wollastonite on proliferation and differentiation of human bone marrow-derived stromal cells in PHBV/ wollastonite composite scaffolds. J Biomater Appl 2009;24:231-46; doi:10.1177/0885328208096043.

21. Saravanan S, Vimalraj S, Vairamani M, Selvamurugan N. Role of mesoporous wollastonite (calcium silicate) in mesenchymal stem cell proliferation and osteoblast differentiation: a cellular and molecular study. J Biomed Nanotechnol 2015;11:1124-38; doi:10.1166/ jbn.2015.2057.

22. Sainitya R, Sriram M, Kalyanaraman V, Dhivya S, Saravanan S, Vairamani M, et al. Scaffolds containing chitosan/carboxymethyl cellulose/mesoporous wollastonite for bone tissue engineering. Int J Biol Macromol 2015;80:481-8; doi:10.1016/j.ijbiomac.2015.07.016.

23. Ramos TL, Sánchez-Abarca LI, Muntión S, Preciado S, Puig N, LópezRuano G, et al. MSC surface markers (CD44, CD73, and CD90) can identify human MSC-derived extracellular vesicles by conventional flow cytometry. Cell Commun Signal 2016;14:1-4; doi:10.1186/ s12964-015-0124-8.

24. Dominici M, Le Blanc K, Mueller I, Slaper-Cortenbach I, Marini $\mathrm{F}$, Krause $\mathrm{D}$, et al. Minimal criteria for defining multipotent mesenchymal stromal cells. The International Society for Cellular Therapy position statement. Cytotherapy 2006;8:315-7; doi:10.1080/14653240600855905.

25. Murphy CM, Haugh MG, O'Brien FJ. The effect of mean pore size on cell attachment, proliferation and migration in collagenglycosaminoglycan scaffolds for bone tissue engineering. Biomaterials 2010;31:461-6; doi:10.1016/j.biomaterials.2009.09.063.

26. Baier T, Dupeux G, Herbert S, Hardt S, Quéré D. Mesoporous calcium silicate compositions and methods for synthesis of mesoporous calcium silicate for controlled release of bioactive agents, US 8,916,198 B2, 2013; doi:10.1016/j

27. Vladkova TG. Surface engineered polymeric biomaterials with improved biocontact properties. Int J Polym Sci 2010;2010:1-22; doi:10.1155/2010/296094.

\section{How to cite this article:}

Macrin D, Narayanan V, Devi A. Osteogenic potential of primary stem cells derived from the human dental pulp is enhanced by carboxymethyl cellulose/chitosan scaffold doped with wollastonite particles. J Appl Biol Biotech 2021; 9(S1):

1-6. 